\title{
Plasma coating of carbon nanofibers for enhanced dispersion and interfacial bonding in polymer composites
}

\author{
Donglu Shi ${ }^{\mathrm{a})}$ \\ Department of Chemical and Materials Engineering, University of Cincinnati, Cincinnati, Ohio 45221 \\ Jie Lian \\ Department of Nuclear Engineering and Radiological Science, University of Michigan, Ann Arbor, \\ Michigan 48109 \\ Peng $\mathrm{He}$ \\ Department of Chemical and Materials Engineering, University of Cincinnati, Cincinnati, Ohio 45221
}

L. M. Wang

Department of Nuclear Engineering and Radiological Science, University of Michigan, Ann Arbor, Michigan 48109

Feng Xiao and Ling Yang

Department of Chemical and Materials Engineering, University of Cincinnati, Cincinnati, Ohio 45221

Mark J. Schulz

Department of Mechanical Engineering, University of Cincinnati, Cincinnati, Ohio 45221

David B. Mast

Department of Physics, University of Cincinnati, Cincinnati, Ohio 45221

(Received 2 June 2003; accepted 31 October 2003)

\begin{abstract}
Ultrathin films of polystyrene were deposited on the surfaces of carbon nanofibers using a plasma polymerization treatment. A small percent by weight of these surface-coated nanofibers were incorporated into polystyrene to form a polymer nanocomposite. The plasma coating greatly enhanced the dispersion of the nanofibers in the polymer matrix. High-resolution transmission-electron-microscopy (HRTEM) images revealed an extremely thin film of the polymer layer $(\sim 3 \mathrm{~nm})$ at the interface between the nanofiber and matrix. Tensile test results showed considerably increased strength in the coated nanofiber composite while an adverse effect was observed in the uncoated composites; the former exhibited shear yielding due to enhanced interfacial bonding while the latter fractured in a brittle fashion. (c) 2003 American Institute of Physics. [DOI: 10.1063/1.1636521]
\end{abstract}

While carbon nanotubes/nanofibers can potentially be used in many applications because of their desirable bulk properties, ${ }^{1-6}$ the surface of the nanotubes/nanofibers, unfortunately, is often not ideal for particular applications. Recently, it has been shown in laboratory scale tests that the physical properties and performance of composite materials can be significantly improved by the addition of small percentages of carbon nanotubes and nanofibers. ${ }^{7-10}$ However, there have not been many successful large scale tests that show the advantage of using nanofibers as fillers over traditional carbon fibers. This problem is associated with dispersing the nanofibers and creating a strong interface between the nanofiber and the polymer matrix..$^{11,12}$ The strong interface between the nanofiber and the polymer matrix is essential to transfer the load from the matrix to the nanofibers and thereby to enhance the mechanical properties of the composite. In addition, the as-produced nanofibers usually form as aggregates that behave differently in response to a load as compared to individual nanofibers. ${ }^{13,14}$ To maximize the advantage of nanofibers as reinforcing particles in high strength composites, the aggregates need to be broken up and dispersed or crosslinked to prevent slippage.

A key aspect of being able to manipulate the properties

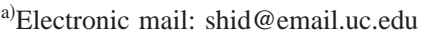

of the nanofibers is the surface treatment of the nanofibers. Plasma polymerization is a method that has been used to deposit an extremely thin film on the surfaces of nanofibers and nanoparticles. ${ }^{15-17}$ In our previous studies we demonstrated the plasma deposition of a thin polymer film on the surfaces of carbon nanotubes. ${ }^{18}$ In this letter, we present results on the microstructure, dispersion, and mechanical properties of a polymer composite impregnated with coated carbon nanofibers. The fracture morphology of both coated and uncoated nanofibers composites have been identified by scanning electron microscopy (SEM) observation using a Philips XL30 FEG SEM. HRTEM images were acquired using a JEOL 2010F TEM to show the interface structures that are responsible for the improved properties. TEM samples of the composite samples were prepared by ultramicrotomy with a cutting thickness of $60 \mathrm{~nm}$.

In this experiment, we used commercial Pyrograf III carbon nanofibers as substrates. ${ }^{18}$ The Pyrograf III nanofibers are $70-200 \mathrm{~nm}$ in diameter, $50-100 \mu \mathrm{m}$ long. ${ }^{19}$ The plasma reactor used for thin film deposition on the nanofibers and the associated processing conditions have been introduced previously. ${ }^{20-22}$ Polystyrene is used as the monomer for the plasma polymerization in the present letter. An ultrathin film showing amorphous features was deposited on both the inner and outer surfaces of the nanofibers after plasma treatment. 

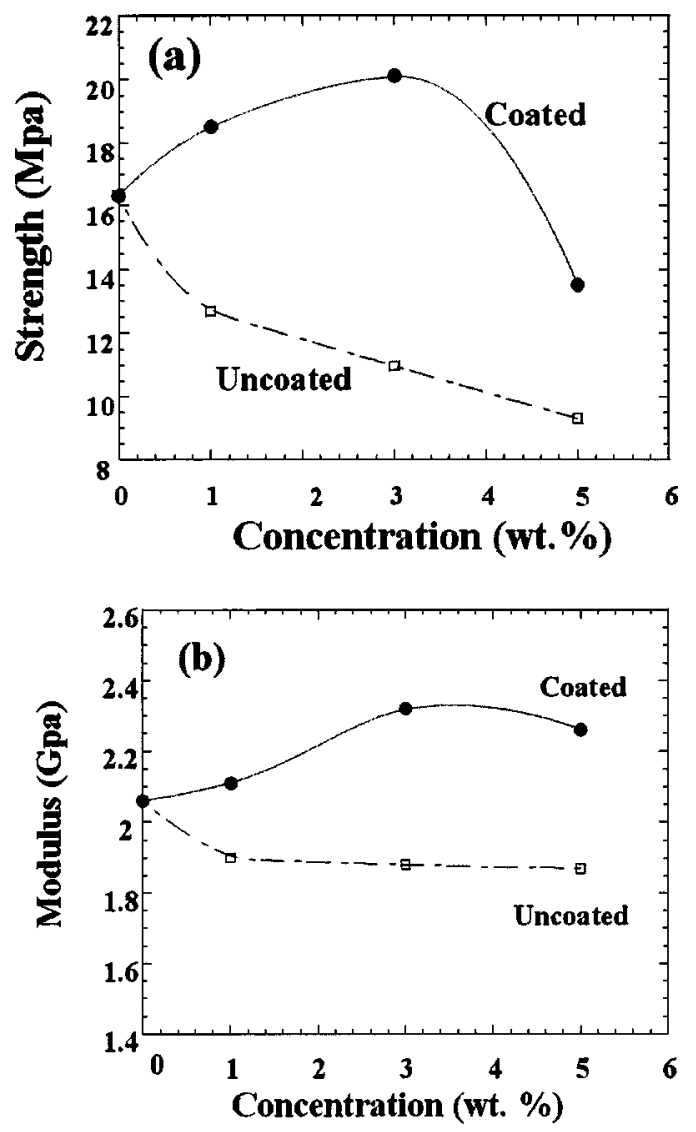

FIG. 1. (a) Strength vs concentration and (b) modulus vs concentration for both coated and uncoated nanofiber composite at the concentration indicated.

The thickness of ultrathin film is approximately $2-7 \mathrm{~nm}$ completely surrounding the nanofiber surfaces. ${ }^{18}$

Two grams of polystyrene powder was mixed mechanically with coated or uncoated nanofibers in appropriate proportions, i.e., $0,1,3$, and $5 \mathrm{wt} \%$. A solvent (50 $\mathrm{ml}$ toluene) was then added to the premixed powers and the powder was thoroughly dispersed ultrasonically. The solution was evapo-
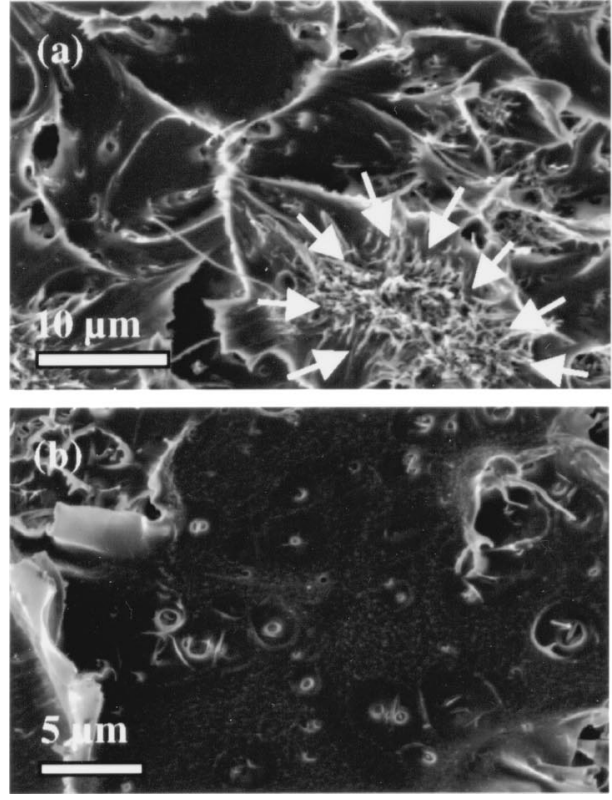

FIG. 2. SEM micrographs of the $3 \mathrm{wt} \%$ uncoated nanofiber-polymer composite showing (a) uncoated nanofiber clusters; (b) flat, brittle type fracture surface; and (c) severe pullouts. A SEM image (d) of the $3 \mathrm{wt} \%$ coated nanofiber composite showing a well dispersed carbon nanofiber in polymer matrix, and the wavy type fracture surface suggesting shear yielding. rated until its volume reduced to $\sim 20 \mathrm{ml}$ (the ultrasonic vibration was kept on during this process) and then poured into an $80 \mathrm{~mm} \times 60 \mathrm{~mm} \times 6.5 \mathrm{~mm}$ aluminum mold. The solution was kept at room temperature and dried for 7 days. After the sample was completely dried, it was sectioned into 50 $\mathrm{mm} \times 6 \mathrm{~mm} \times 0.4 \mathrm{~mm}$ samples for tensile testing according to the ASTM D 822-97: "Standard Test Method for Tensile Properties of Thin Plastic Sheeting."

An Instron mechanical testing machine, model 2525818 , with a $1 \mathrm{~mm} / \mathrm{min}$ crosshead speed was used for the tensile test. Figure 1(a) shows the strength as a function of nanofiber concentration for both coated and uncoated nanofiber composites. For the uncoated nanofiber composite, the strength of the composite decreases gradually as the nanofiber concentration increases; while the coated counterpart showed a significant increase in strength. The maximum strength of the coated nanofiber composite takes place at 3 wt $\%$ and then gradually decreases up to $5 \mathrm{wt} \%$. A similar trend is seen in the modulus value [Fig. 1(b)], which is consistent with that of strength value. The decrease in properties above $3 \mathrm{wt} \%$ loading may be due to the nanofibers not being initially as well dispersed, and due to later agglomeration of the nanofibers in the matrix.

In the $3 \mathrm{wt} \%$ uncoated sample, the nanofibers are highly clustered in the matrix with approximately a $\sim 10 \mu \mathrm{m}$ diameter [Fig. 2(a)], as indicated by the arrows. These clusters appear to be densely distributed with a small spacing of $\sim 25$ $\mu \mathrm{m}$. Another important characteristic of the uncoated nanofibers composite is the rather flat fracture surface [Fig. 2(b)] indicating the nature of brittle fracture. At these fracture surfaces severe pullouts of nanofibers are also observed [Fig. 2(c)]. In sharp contrast, the dispersion is greatly improved in the coated nanofibers composite and the coated nanofibers are well dispersed in the matrix with a wavy type of fracture surface morphology [Fig. 2(d)]. The interface structure between the CNTs and polymer matrix was studied by HRTEM for both coated [Fig. 3(a)] and uncoated nanofiber [Fig. 3(b)]
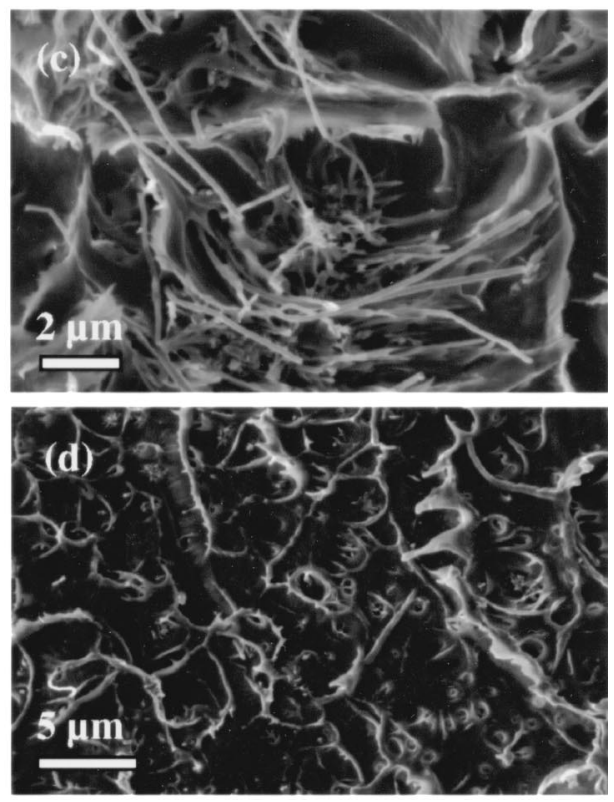


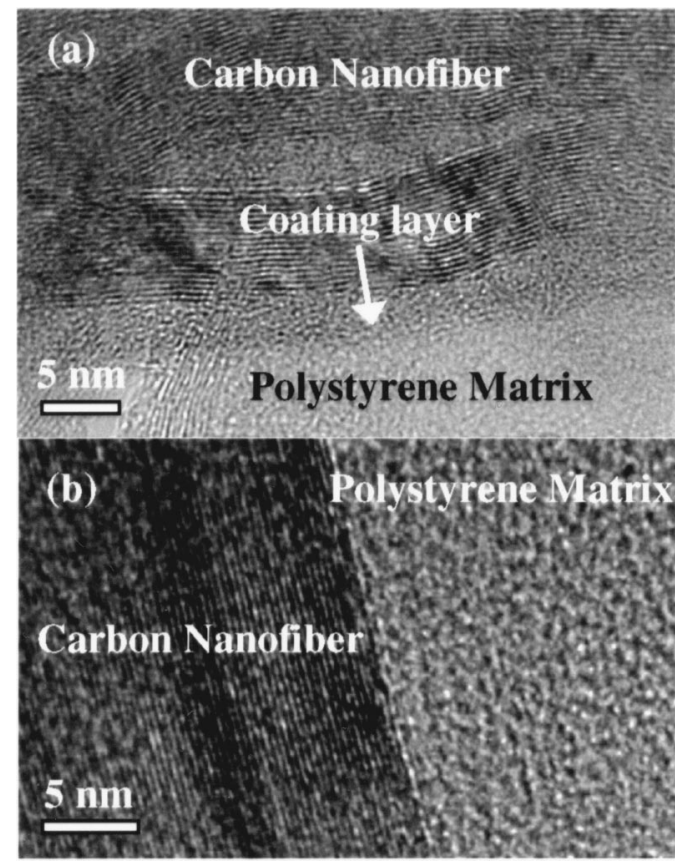

FIG. 3. HRTEM images of (a) interface of coated nanofibers with matrix and (b) interface of uncoated nanofiber with matrix.

composite samples. The contrast in Fig. 3(a) clearly shows the coating layer between the carbon nanofiber and the matrix, whereas the uncoated carbon nanofiber surface is in direct contact with the matrix as shown in Fig. 3(b).

The central focus of this study is on the enhanced interfacial bonding due to plasma coated thin films on nanofibers. The nature of strengthening in nanofiber-reinforced composites is dependent on the stress transfer between the matrix and nanofiber. For polymers, tensile loading can produce matrix cracking, nanofiber bridging, nanofiber rupture, nanofiber pullout, and debonding. In this experiment, pullouts of nanofibers were observed in the uncoated nanofiber composite as indicated in Fig. 2(c), especially within the cluster regions. As the nanofibers are clustered, the interface area between the matrix and nanofiber is greatly reduced leading to significantly lowered strength. Furthermore, these clusters act as large voids that are responsible for decreasing the strength of the composite as the nanofiber concentration increases (Fig. 1).

As the nanofiber surfaces are modified by plasma coating, the surface energy can be significantly lowered, which can enhance dispersion in the polymer matrix. The welldispersed nanofibers in the matrix appear to have few clusters and pullouts. In addition, the adhesive film on the nanofiber surface, as shown in Fig. 3(a), can provide enhanced bonding, and therefore contribute to a considerably increased strength in the coated-nanofiber composite. The efficiency of stress transfer is strongly dependent on the maximum value of the shear stress acting at the interface. This stress is also characterized as the interfacial shear strength that depends on the nature of bonding at the inter- face. As indicated by the interface HRTEM, there is clearly an interfacial adhesion layer due to the coated polymer film on the nanofiber surface. Although a quantitative measure $^{23,24}$ of the interfacial shear strength has not been conducted, the effect of enhanced bonding is evident from the increased composite strength and fracture surface morphology.

In summary, an approach has been developed to enhance the dispersion and interfacial bonding of nanofibers in polymer composites. As a result of plasma coating, carbon nanofibers can be well dispersed in a polymer matrix. Both the fracture behavior and tensile strength data indicate that the well-dispersed nanofibers have contributed to enhanced interfacial shear strength, and therefore have increased the overall strength of the material.

This research was supported in part by Air Force Contract No. F33615-01-D-5802.

${ }^{1}$ S. Iijima, Nature (London) 354, 56 (1991).

${ }^{2}$ R. H. Baughman, C. Cui, A. A. Zakhidov, Z. Iqbal, J. N. Barisci, G. M. Spinks, G. G. Wallace, A. Mazzoldi, D. De Rossi, A. G. Rinzler, O. Jaschinski, S. Roth, and M. Kertesz, Science 284, 1340 (1999).

${ }^{3}$ M. Gao, L. Dai, R. H. Baughman, G. M. Spinks, and G. G. Wallace, Electroactive Polymer Actuators and Devices, SPIE Proceedings, 2000, p. $18-24$.

${ }^{4}$ V. G. Hadjiev, M. N. Iliev, S. Arepalli, P. Nikolaev, and B. S. Files, Appl. Phys. Lett. 78, 3193 (2001)

${ }^{5}$ C. Liu, H. M. Cheng, H. T. Cong, F. Li, G. Su, B. L. Zhou, and M. S. Dresselhaus, Adv. Mater. (Weinheim, Ger.) 12, 1190 (2000).

${ }^{6}$ D. A. Walters, M. J. Casavant, X. C. Qin, C. B. Huffman, P. J. Boul, L. M. Ericson, E. H. Haroz, M. J. O'Connell, K. Smith, D. T. Colbert, and R. E. Smalley, Chem. Phys. Lett. 338, 14 (2001).

${ }^{7}$ J. Zhu, J. D. Kim, H. Q. Peng, J. L. Margrave, V. N. Khabashesku, and E. V. Barrera, Nano Lett. 3, 1107 (2003).

${ }^{8}$ E. T. Tostenson and T. W. Chou, J. Phys. D 35, L-77 (2002).

${ }^{9}$ M. Cadek, J. N. Coleman, V. Barron, K. Hedicke, and W. J. Blau, Appl. Phys. Lett. 81, 5123 (2002).

${ }^{10}$ S. J. V. Franklanda, V. M. Harikb, G. M. Odegarda, D. W. Brennerc, and T. S. Gatesd, Compos. Sci. Technol. 63, 1655 (2003).

${ }^{11}$ K.-T. Lau, Chem. Phys. Lett. 370, 399 (2003).

${ }^{12}$ A. A. Mamedov, N. A. Kotov, M. Prato, D. M. Guldi, J. P. Wicksted, and A. Hirsch, Nat. Mater. 1, 190 (2002).

${ }^{13}$ P. M. Ajayan, Chem. Rev. (Washington, D.C.) 99, 1787 (1999).

${ }^{14}$ P. M. Ajayan and O. Z. Zhou, in Carbon Nanotubes, edited by M. S. Dresselhaus and Ph. Avouris (Springer, Berlin, 2001).

${ }^{15}$ C. Bayer, M. Karches, A. Matthews, and P. R. Von Rohr, Chem. Eng. Technol. 21, 427 (1998).

${ }^{16}$ S. Eufinger, W. J. van Ooij, and T. H. Ridgway, J. Appl. Polym. Sci. 61, 1503 (1996).

${ }^{17}$ W. J. van Ooij, S. Eufinger, and T. H. Ridgway, Plasma and Polymers 1, 231 (1996).

${ }^{18}$ D. Shi, J. Lian, H. Peng, L. M. Wang, W. J. Van Ooij, M. Schulz, Y. J. Liu, and D. B. Mast, Appl. Phys. Lett. 81, 5216 (2002).

${ }^{19}$ Applied Sciences Inc., www.apsci.com.

${ }^{20}$ D. Shi, S. X. Wang, W. J. van Ooij, L. M. Wang, J. G. Zhao, and Z. Yu, Appl. Phys. Lett. 78, 1243 (2001).

${ }^{21}$ D. Shi, S. X. Wang, W. J. van Ooij, L. M. Wang, J. G. Zhao, and Z. Yu, J. Mater. Res. 17, 981 (2002).

${ }^{22}$ D. Shi, P. He, J. Lian, L. M. Wang, and W. J. van Ooij, J. Mater. Res. 17, 2555 (2002).

${ }^{23}$ C. A. Cooper, S. R. Cohen, A. H. Barber, and H. D. Wagner, Appl. Phys. Lett. 81, 3873 (2002).

${ }^{24}$ A. H. Barber, S. R. Cohen, and H. D. Wagner, Appl. Phys. Lett. 82, 4140 (2003). 